\title{
Livestock Poisoning With Pyrrolizidine-Alkaloid- Containing Plants (Senecio, Crotalaria, Cynoglossum, Amsinckia, Heliotropium, and Echium spp.)
}

\section{By Bryan L. Stegelmeier, Dale R. Gardner, and T. Zane Davis}

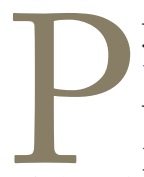
yrrolizidine alkaloids (PAs) are potent liver toxins that have been identified in over 6,000 plants worldwide. Alkaloids are nitrogen-based compounds with potent biological activity. About half of the identified PAs are toxic, with several causing cancer (carcinogenic). PA-containing plants are the most common poisonous plants affecting livestock, wildlife, and humans. Many PA-containing plants are imported weeds that invade pastures and fields. Smaller numbers are native plants that may increase on ranges or disturbed areas. Most are unpalatable, becoming a problem for livestock only when alternative forages are unavailable or included in hay and other harvested feeds. Human poisoning is most often a result of contaminated grain or flour, although recent poisonings are linked to the use of PAs containing herbal preparations. This paper briefly introduces high-risk PA-containing plants in North America and summarizes their toxicity and subsequent pathology. We also review current treatments and strategies to avoid losses to PA poisoning.

Tansy ragwort (Senecio jacobaea) is a common western European plant inadvertently introduced into Eastern Europe, South Africa, Australia, New Zealand, and northwestern regions of North America. As with most PAcontaining plants, tansy is unpalatable and generally not eaten by livestock. Poisoning occurs when plants or seeds contaminate feeds, when grazing animals cannot easily differentiate the early rosette from grasses and clovers, or when other forages are unavailable. The lethal dose for cattle is about 3-4 pounds of fresh plant per day for several weeks. Tansy ragwort also can contaminate feeds when it is harvested and included in hays.
Riddell's groundsel (Senecio riddellii) is found in Nebraska, New Mexico, Texas, Colorado, and Wyoming (Fig. 1). Alkaloid concentrations in plants vary greatly, with measured concentrations ranging between $0.2 \%$ and $18.0 \%$ (dry weight). Riddell's groundsel is toxic to cattle, and doses of less than 4 ounces $(0.5 \mathrm{~g})$ of fresh plant per day are toxic to an adult cow. The potentially large PA concentrations increase the risk of poisoning.

Threadleaf or woolly groundsel (Senecio douglasii var. longilobus) is often found on abused or degraded arid rangelands. Found in several southwestern states, its alkaloid concentrations vary from $0.63 \%$ to $2.02 \%$ of the plant dry weight. Doses of just over 1 pound $(2.5 \mathrm{~g})$ of green plant per day for 15 days will poison a cow.

Crotalaria species are of low risk for open range because they are primarily introduced species that are often found along fence rows and ditch banks. Most have long, kidneyshaped seeds that rattle in mature dry pods, resulting in the common name "rattle pod." Their seeds are often harvested with grains, thus contaminating an assortment of foods. Arrowhead rattlebox (Crotalaria sagittalis) ingestion causes Missouri "Bottom disease," in which animals become slow, emaciated, weak, and stuporous with progressive liver degeneration. Most animals die within a few weeks. Horses are highly susceptible and are often poisoned in arrowheadrattlebox-infested stubble fields. Crotalaria contamination of grain has poisoned both livestock and poultry.

Hound's tongue (Cynoglossum officinale), an annual or biennial European plant, is a noxious weed found in many western pastures, rangelands, and fields. Generally unpalatable to livestock, cattle and horses are poisoned when they 


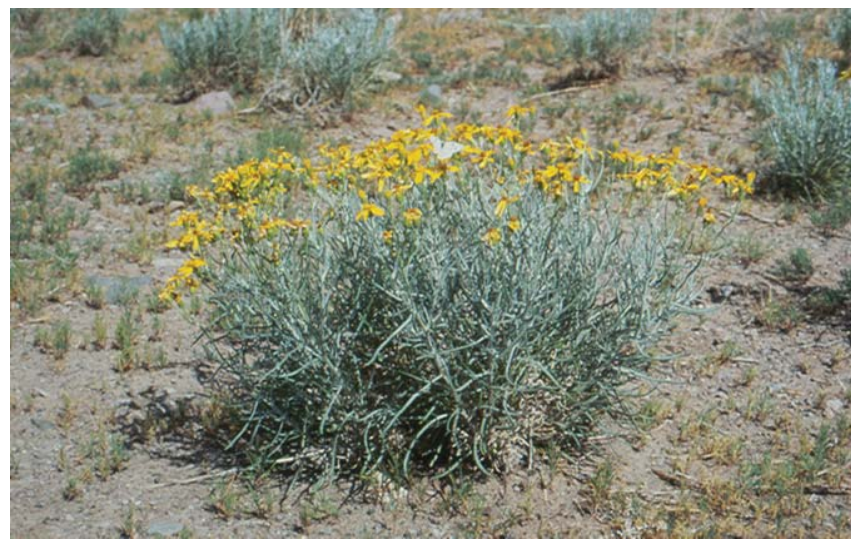

Figure 1. Theadleaf or woolly groundsel (S. douglasii var. longilobus) in flower.

are fed contaminated feed. PA concentrations are greatest in immature plants, and about 1 pound $(2.5 \mathrm{~g})$ of green plant for 14 days would be lethal for cows and horses.

Fiddlenecks (Amsinckia spp.) are annuals that include nearly 50 native species in the Americas and Europe (Fig. 2). Fiddleneck populations often expand in disturbed areas or degraded rangelands. They generally contain relatively small amounts of a mixture of PAs, most of which have low or moderate toxicity. As a result, poisoning is infrequent, and some animals seem to eat fiddlenecks with impunity. However, poisoning does occur, and horses seem to be most susceptible. More work is needed to better define fiddleneck toxicity and determine risks to livestock.

Species within the genera Echium and Heliotropium intermittently poison Australian livestock. Both are found in North America, but incidences of poisoning are rarely reported. These plants have abundant, long-lived flowers, and recent Australian studies show Echium PAs contaminate honey and poison wildlife. There are many other toxic PA-containing plants including comfrey (Symphytum officinale) that are of concern because they are often included

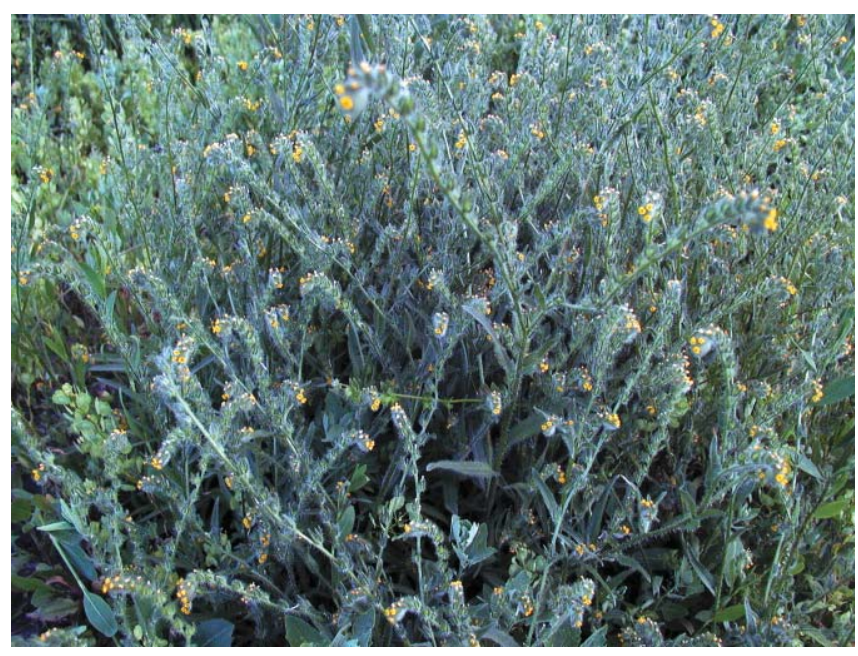

Figure 2. Fiddleneck (Amsinckia intermedia) in flower. in herbal products, but these are rarely a problem on rangelands.

PAs themselves are not toxic but become toxic following bioactivation, or a conversion to the toxic form known as pyrroles. In tissues, pyrroles are highly reactive and bind with adjacent nucleic acids or proteins. The resulting pyrrole-tissue complexes (called adducts) result in loss of cellular function and in some cases cause genetic damage that may result in cancer. Some pyrrole adducts may persist for months or years, with little being known about their ultimate fate. The progressive nature of PA-induced liver damage suggests pyrrolic adducts are recycled, causing repeated tissue damage.

Susceptibility to poisoning is influenced by species, age, sex, and other temporary factors such as biochemical, physiologic, and nutritional status. Animal species also differ in their susceptibility to PAs. For example, under some conditions sheep can consume 20 times the dose of some toxic plants known to kill cattle. For this reason sheep and goats are used to graze pastures dangerous to horses and cattle. Horses appear to be as sensitive to PA toxicity as cattle, with pigs being the most sensitive species. Age and gender also play a large role in determining an animal's response to PAs. Young animals are generally more susceptible than adults. Neonatal and nursing animals and humans develop fatal liver disease while their pregnant or lactating mothers are unaffected. Males are often more susceptible than females. Plant palatability and amount eaten varies with season, location, weather, and the availability of associated forages. PA concentrations also vary with environment, plant phenotype, and site. Usually plants are most toxic in the early bud and flower stages, yet it is difficult to predict PA concentrations across time.

Severity of microscopic lesions (damaged tissue) resulting from PA toxicosis is dose dependent and characterized as acute or chronic. Large PA doses ingested quickly induce acute intoxication characterized by extensive damage and death (necrosis) of liver cells, hemorrhaging, and minimal inflammation. These animals show signs of acute liver failure, including anorexia, depression, jaundice, visceral edema, and fluid in the abdominal cavity. Blood tests will show increased enzyme activity (AST, SDH, ALK, and GGT) as enzymes leak from damaged cells. Also many liver metabolites in the blood (bilirubin and bile acids) increase with impaired liver function. Chronic poisoning is produced when animals are exposed to lower PA doses over a longer duration. Clinical symptoms are not immediately apparent because animals develop only transient elevations in serum enzyme activities. With time, damaged liver cells become abnormally large. Animals may show no clinical signs, and blood enzyme activities may be normal for several months or even years after PA ingestion. However, liver damage may continue, resulting in continued damage with subsequent inflammation, fibrosis, and ultimately cirrhosis. With loss of liver function, chronically poisoned animals 
often do poorly. When subjected to normal stresses such as pregnancy or lactation, they develop clinical liver failure. Additional clinical signs in these cases include photosensitivity, jaundice, or increased susceptibility to other liver diseases such as fatty liver or ketosis. Because disease often develops months after PA exposure, it is difficult to identify offending plants or feed contaminants.

Because clinical signs of poisoning can be delayed, it is often difficult to document exposure to PA-containing plants. Many diagnoses are made using microscopic changes in tissue alone (liver necrosis, fibrosis, proliferation of bile ducts, and abnormally enlarged liver cells). Unfortunately these lesions are nonspecific because they can be produced by other causes. The ubiquitous nature of PA-containing plants and seeds (including PA-contaminated grains) suggests that PA intoxication is underdiagnosed. Chemical and immunologic assays to screen and analyze feed and food for PA contamination are available. Less sensitive methods exist to detect tissue-bound pyrroles (PA metabolites) in animal tissues. Although these have proven to be useful in identifying exposed animals, they lack sensitivity and show only presence or absence of PA metabolites, not tissue concentrations. Work is continuing to improve these diagnostic techniques.

The progressive nature of chronic PA intoxication suggests that low, chronic PA exposure has cumulative effects. Little is known about what doses or durations are damaging, or the effect of subclinical intoxication on growth or production. Ongoing research is developing better diagnostics to monitor PA metabolites and predict development of liver disease. Additional studies are designed to determine the effects of chronic, low-dose exposures and the carcinogenic potential of specific PAs.

In conclusion, none of the various treatments or diet supplements is effective for treating PA intoxication in livestock. Poisoned animals showing clinical symptoms rarely recover, making prevention the best control. Because most poisonings are attributed to contamination of harvested feeds, careful feed inspection is recommended. Contaminated feeds should be discarded or fed to less susceptible species. Inspection of fields before harvest provides the best opportunity to detect PA-containing plants. Though most PA-containing plants are not highly palatable, if possible these plants should be eliminated from pastures and rangelands. Species-specific herbicide regimens are available from county weed and extension services for most plants.

Authors are Veterinary Pathologist, bryan.stegelmeier@ars.usda. gov (Stegelmeier), Research Chemist (Gardner), and Research Biochemist (Davis), Poisonous Plant Research Laboratory, Agricultural Research Service, United States Dept of Agriculture, 1150 E 1400 N, Logan, UT 84341, USA. Published with the approval of the Director as Journal Paper No. 8039 of the Utah Agricultural Experiment Station. 